\title{
Simply partitioned DP Matching and Threshold Equalizing in DWT Domain On-line Signature Verification
}

\author{
Isao Nakanishi, Shingo Nakatani and Shota Koike* \\ Graduate School of Engineering \\ Tottori University \\ 4-101 Koyama-minami, Tottori, 680-8552 Japan \\ nakanishi@ele.tottori-u.ac.jp
}

\begin{abstract}
In this paper, we introduce simply partitioned DP (dynamic programming) matching and threshold equalizing into the verification process in DWT (discrete wavelet transform) domain on-line signature verification in order to improve the performance. The simply partitioned DP matching divides both data series (verification and template data) into several partitions and calculate sub DP distance every partition. Even if mismatched pairs are caused in each partition, they are initialized at the beginning of the next partition and matching errors could be reduced. The threshold equalizing suppresses the variation range of optimal thresholds for all users (signatures), so that it prevents the verification performance from degrading by common use of single threshold for all users. In particular, we propose two equalizing methods in which the relation between the number of sampled data and optimal thresholds in signatures are approximated and adjusted by linear and nonlinear functions. In verification experiments using the signature database: SVC2004, it is confirmed that the proposed methods are effective for improving the performance.
\end{abstract}

Keywords-biometrics; on-line signature verification; DWT; DP matching; threshold equalizing

\section{INTRODUCTION}

Biometrics attracts attention since person authentication becomes very important in networked society. As the biometrics, fingerprint, iris, face, ear, vein, gate, voice, and signature are well known and they are used in various applications [1]. Especially, assuming the mobile access using a portable terminal such as a personal digital assistant (PDA), a camera, microphone, and pen-tablet are normally equipped; therefore, the authentication using a face, voice and/or signature can be realized with no additional sensor.

On the other hand, the safety of biometric data is discussed actively. Every human being has limited biometrics, for example, only ten fingerprints and one face. If the biometric data are leaked out and it is known whose they are, they are never used for authentication again.

To deal with this problem, cancelable biometric techniques have been proposed, which use not biometric data directly but one-to-one transformed data from the biometric

* Presently at the Daikin Industries, Ltd., Osaka, Japan data. However, such a technique is unnecessary if the biometrics itself is cancelable.

Among various biometrics, only on-line signature is cancelable. Even if signature shape is known by others, it is possible to cope with the problem by changing the shape. In the on-line signature, the habit of writing is biometrics and it is not remained in the signature shape; therefore, to imitate it is quite difficult even if the signature shape is copied.

We have proposed a new on-line signature verification method in which pen-position parameter is decomposed into sub-band signals using the discrete wavelet transform (DWT) and total decision is done by fusing the verification results in sub-bands [2], [3]. Since signature shapes are visible, it is relatively easy to forge the pen-position parameter by tracing genuine signatures by others. However, assuming to use the PDA, detecting functions of other parameters such as pen-pressure, pen-altitude, and/or pen-direction are not equipped and so only the pen-position parameter is available. Even if the signature shape is forged, individual features of the signature are enhanced and extracted in the sub-band signals, so that the proposed method can verify such a wellforged signature.

In verification process of the proposed method, the dynamic programming (DP) matching is adopted to make it possible to verify two data series with different number of sampled points. The purpose of the DP matching is to find the best combination between such two data series. Concretely, the DP distance is calculated in every possible combination of two data series and as a result the combination which has the smallest DP distance is regarded as the best. However, there might be many mismatched pairs in the matching. It results in excessively large DP distance even though both data series are of genuine signatures. In this paper, we propose simply partitioned DP matching in order to reduce the errors by the mismatched pairs.

Moreover, we also introduce threshold equalization into the verification process. The DP distance is proportional to the number of signature's sampled data, that is, signature complexity (shape), so that if it is used as the criterion in the verification, each signature has a different threshold. 


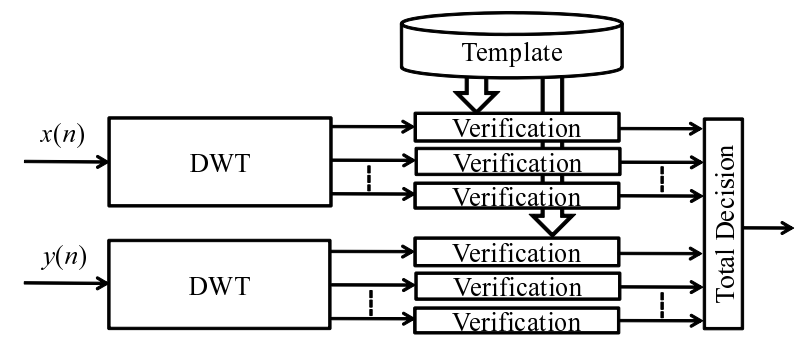

Figure 1. DWT domain on-line signature verification.

However, it is general to use single threshold commonly in an authentication system. If the common threshold is used for all signatures, it results in the degradation of verification performance. We have studied threshold equalization in the on-line signature verification [4], [5]. In this paper, we propose new equalizing methods based on linear and nonlinear approximation between the number of sampled data and optimal thresholds.

\section{DWT DOMAIN ON-LINE SIGNATURE VERIFICATION}

Figure 1 shows the signal flow diagram of the proposed DWT domain on-line signature verification. An on-line signature is captured as $x$ and $y$ coordinate data in a digital pen-tablet system. Their sampled data: $x(n)$ and $y(n)(n=$ $\left.0,1, \cdots, S_{\text {num }}-1\right)$ are respectively decomposed into subband signals based on the DWT. At the verification stage, each decomposed signal is compared with its template which is memorized in advance and then similarity is evaluated at each sub-band and it results in a score. A final score is obtained by combing the scores at all sub-bands and both coordinates. Finally, total decision is done by comparing the final score with a threshold and it is verified whether the signature data are of genuine.

The reason why the sub-band decomposition enhances the difference between signatures is as follows. Each signature is digitized using the pen-tablet system at equal (common) sampling period. However, writing time of all signatures is normalized in order to suppress intra-class variation as shown in Fig. 2. Concretely, the sampling period of each signature is divided by the number of sampled data and it results in the real-valued sampling period in $0 \sim 1$. Each signature has different number of sampled data and thereby has the different normalized sampling period, that is, the different sampling frequency.

Sub-band decomposition of the DWT corresponds to an octave-band filter bank and its maximum frequency is determined by the sampling frequency. In the octave-band filter bank, the whole frequency band is divided into low and high bands and this decomposition is repeated for the lower frequency band.

In general, variation of the writing time in genuine signatures is small, so that their sampling frequencies are

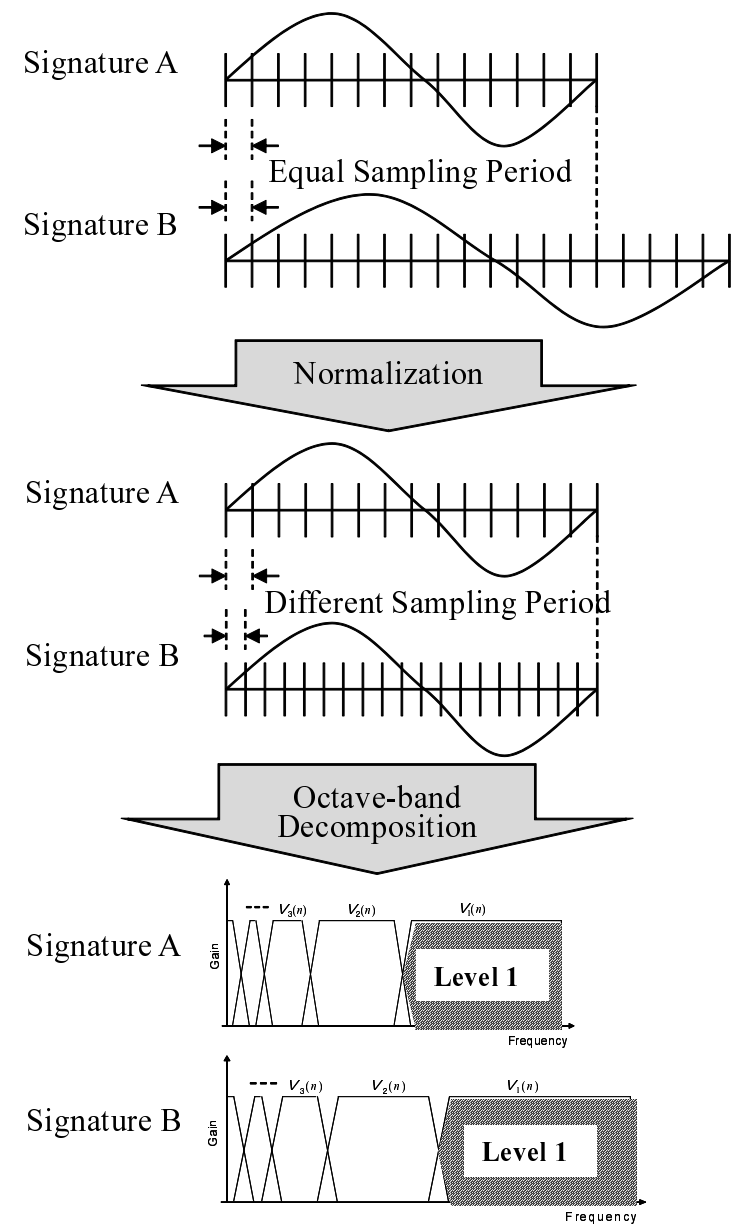

Figure 2. Effect of Sub-band Decomposition.

comparable. On the other hand, in the case of forged signatures, the variation of the writing time is large since it is not easy for forgers to imitate writing speed and rhythm of the genuine signatures. Resultingly, sampling frequencies of the forged signatures become greatly different from those of the genuine signatures.

If the sampling frequencies are different, each octave band (decomposition level) includes different frequencies. In other words, even if the levels are the same, actual frequencies included in the levels are different as illustrated in Fig. 2.

Thus, the sub-band decomposition is effective for accentuating the difference between genuine signatures and forged ones. Of course, if the forgers imitate writing speed and rhythm of genuine signatures, it is impossible for the proposed method to distinguish forged signatures from genuine ones.

On the other hand, verification of signatures is performed every stroke (intra-stroke or inter-stroke) in order to deal with the problem that one-to-one matching cannot be applied in the verification since the on-line signature has large 


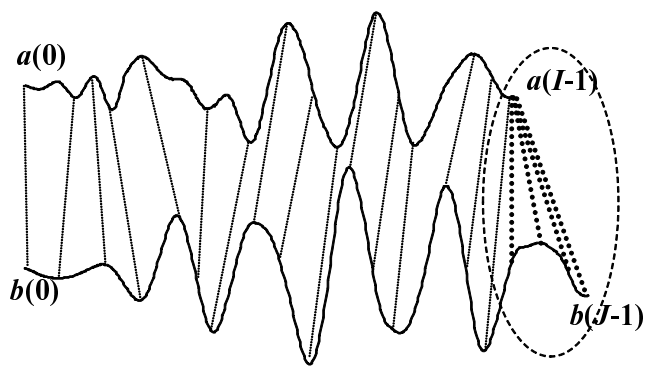

Figure 3. Mismatched pairs in DP matching.

intra-class variation. The details are omitted due to space limitation. Please refer to Ref. [2], [3]. However, a part of signature databases eliminates the data in inter-strokes. The conventional method could not be applied to such a database directly.

\section{DP MATCHING}

The DP matching is effective on verification even when two data series compared have different number. To introduce the DP matching into the verification process enables to apply the DWT domain on-line signature verification to standard databases.

The purpose of the DP matching is to find the best combination between the two sampled data series. Letting the two series be $a(i)(i=0,1, \cdots, I-1), b(j)(j=0,1, \cdots, J-1)$, the local distance at $k$ th is defined as

$$
d(k)=\left|a(i)_{k}-b(j)_{k}\right|(k=0,1, \cdots, K-1)
$$

where instead of $i$ and $j, k$ is used as another index since both the data are permitted to be referred redundantly.

By accumulating such distances in one possible combination between the two series, DP distance is given by

$$
D(a, b)=\sum_{k=0}^{K-1} w(k) d(k)
$$

where $w(k)$ is a weighting factor. After calculating the DP distance in all possible combinations, we can find the best combination by searching the combination with the smallest DP distance.

Moreover, since the DP distance depends on the number of sampled data, the normalized DP distance is used in general.

$$
D(a, b)=\left(\sum_{k=0}^{K-1} w(k) d(k)\right) /(I+J)
$$

\section{A. Simply partitioned DP matching}

Figure 3 shows an example of the DP matching, where both $a(i)$ and $b(j)$ are genuine signature data but there are mismatched pairs circled with the broken line. The mismatched pairs bring large accumulative errors and it

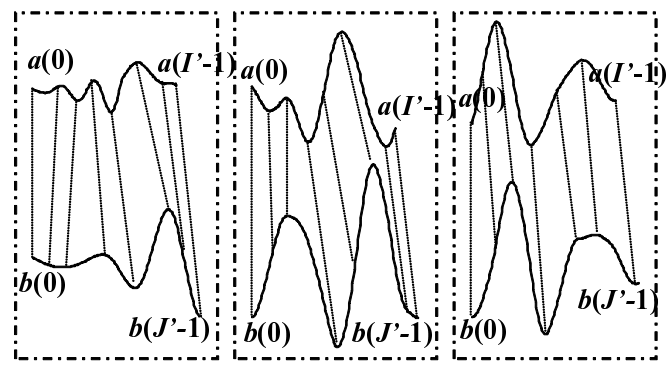

Figure 4. Simply partitioned DP matching.

results in excessively large DP distance even though the two series are similar.

In this paper, we propose to divide both data series into several partitions of the same number and calculate sub DP distance every partition. Total DP distance is obtained by summing the sub DP distances in all partitions. The partitioning is performed by dividing the number of data by the same integer number. If the division leaves remainders, they are singly distributed to partitions.

The concept of the proposed simply partitioned DP matching is illustrated as Fig. 4, where the number of partitions is 3. Even if mismatched pairs are caused in each partition, accumulative errors are initialized at the beginning of the next partition and as a result, it prevents the DP distance from becoming excessively large.

By the way, the matching window $(|i-j| \leq R)$ is generally adopted to exclude unlikely pairs as shown in Fig. 5 (a). It is comparatively effective for reducing false acceptance of others' signatures. On the other hand, the proposed simply partitioned DP matching is depicted in (b) and it is clear that it is more effective for excluding unlikely pairs than the matching window. In addition, assuming that genuine signatures have comparable writing speed and rhythm, it is expected that optimal pairs in two genuine signatures verified exit diagonally. As a result, to constrain pairing in diagonal partitions is effective for reducing not only false acceptance of the forged signatures but also false rejection of the genuine signatures.

Let the number of partitions be $Q$, the sub DP distance in each partition at sub-band level $l$ is given by $D(a, b)_{l}^{q}$ and the DP distance is obtained by summing the sub DP distances in all partitions.

$$
D(a, b)_{l}^{x, y}=\left(\sum_{q=1}^{Q} D(a, b)_{l}^{q}\right) /(I+J)
$$

where the DP distance from $x$ or $y$ coordinate is represented using $x, y$.

As the final score, total DP distance is given by

$$
T D=c_{x} \cdot \frac{1}{L} \sum_{l=M-L+1}^{M} D(a, b)_{l}^{x}+c_{y} \cdot \frac{1}{L} \sum_{l=M-L+1}^{L} D(a, b)_{l}^{y}
$$




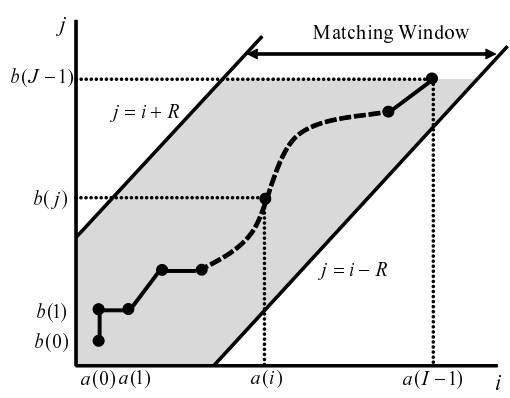

(a)

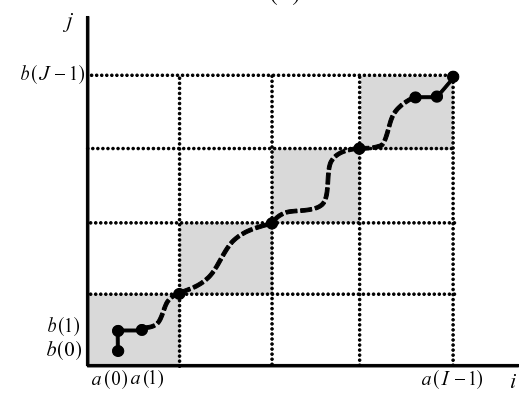

(b)

Figure 5. Matching window and simply partitioned DP matching.

where $c_{x}$ and $c_{y}$ are weights for combining the DP distances from $x$ and $y$ coordinates and $c_{x}+c_{y}=1, c_{x}>0, c_{y}>$ $0 . M$ is the maximum number of sub-bands, that is, the decomposition level and $L$ is the number of levels used in the total decision.

Such a concept that the mismatched pairs were reduced by partitioning the DP distance had been already proposed in Ref. [6], [7] but they assumed to print Chinese characters and the partitioning was done every one character. Therefore, it could not be directly applied to the case of connected characters as a signature.

\section{B. Experiments}

In order to confirm the effectiveness of the simply partitioned DP matching, we carried out experiments in the following conditions.

The decomposition level $M$ was 8 and the number of levels used in the final decision stage $L$ was 4 . The combination weights were $c_{x}=0.5$ and $c_{y}=0.5$, which equivalent to averaging. Templates were generated by ensemble-averaging five genuine signatures.

We used part of the on-line signature database: SVC2004 [8] in which the data in inter-strokes were eliminated. The number of subjects was 40 and 17 subjects signed their names in Chinese characters and the rest in alphabetical ones. For collecting skilled forgeries, imposters could see genuine signatures that they would attempt to forge. The total number of signatures was 1600 . Please refer to Ref. [8] for more information.
Verification performance was evaluated by using the equal error rate (EER) where a false rejection rate (FRR) was equal to a false acceptance rate (FAR). The EERs according to the number of partitions are summarized in Table I. From

Table I

EERS ACCORDING TO THE NUMBER OF PARTITIONS.

\begin{tabular}{|c|c|c|c|c|c|c|}
\hline NP & 0 & 2 & 3 & 4 & 5 & 6 \\
\hline EER (\%) & 20.0 & 17.8 & 16.4 & 16.6 & 17.0 & 16.4 \\
\hline
\end{tabular}
NP: Number of Partitions

these results, it is confirmed that to introduce the simply partitioned DP matching decreased the EER by $2-3 \%$.

\section{THRESHOLD EQUALIZING}

There is another important problem for introducing the DP matching into our verification method. In not only the on-line signature verification but also all biometric authentication systems, the final score is compared with a threshold which is preliminary determined. In addition, the threshold should be common to all users (signatures). However, the final score is proportional to the DP distance and when the DP distance of each signature is greatly different from those of others, verification performance tends to be degraded by using the common threshold.

In general, the normalized DP distance given by Eq. (3) is used for dealing with this problem. However, the normalization also makes the DP distances of forged signatures small and thereby might increases the FAR.

By the way, assuming that the thresholds for all signatures were optimal, the mean value of all EERs was $14.8 \%$ even using unnormalized DP matching while EER was $20.0 \%$ by the normalized DP matching at zero partition in the previous experiment. This suggests that if the threshold is not normalized but optimal for all signatures, the verification performance is improved further.

We have studied threshold equalizing [4], [5]. In the online signature, a complex signature has large number of sampled data. On the other hand, the complex signature has larger intra-class variation and so it makes the DP distance large in general. Final decision is done by comparing the DP distance with a threshold; therefore, to make the DP distance inversely proportional to the number of sampled data suppresses the variation range of the DP distance and then it leads to the equalization of the threshold.

Based on the above concept, the conventional equalization is defined as

$$
T D_{e q}^{p}=\frac{\gamma}{S_{n u m}^{p}} T D^{p}
$$

where $p$ is user number and $T D^{p}, S_{n u m}^{p}$, and $T D_{e q}^{p}$ are respectively the DP distance, the number of sampled data, and final (adjusted) DP distance of each user. $\gamma$ is a constant for adjusting the final DP distance to an appropriate value. When the number of sampled data in a signature is too small, 


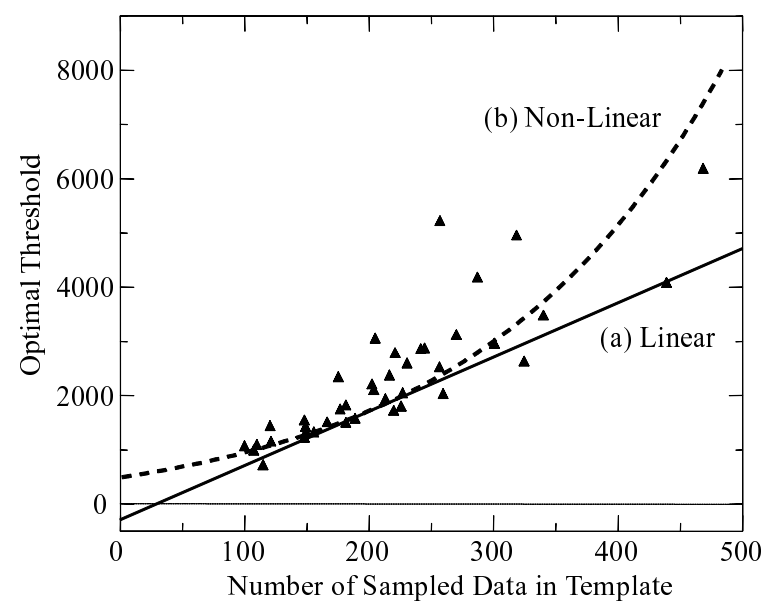

Figure 6. Relation between the number of sampled data and optimal thresholds.

the DP distance of the signature is enlarged. On the other hand, large number of sampled data in a signature reduces the DP distance.

\section{A. New threshold equalizing method}

Figure 6 shows the relation between the number of sampled data in signatures (templates) and their optimal thresholds using 4 partitioned DP matching in SVC2004. The optimal threshold is defined by the threshold to obtain the EER. It is confirmed that the relation between the number of sampled data and the threshold is not simple as assumed in the conventional equalization. In particular, since the optimal thresholds are widely distributed, it is easy to guess that the common use of single threshold is not good for verification performance.

1) Equalization using linear approximation: Assuming that the relation between the number of sampled data and the optimal threshold (the DP distance) is approximated by a linear function as the solid line (a) shown in Fig. 6, the DP distances are equalized as

$$
T D_{e q}^{p}=\frac{\gamma}{\alpha \cdot S_{n u m}^{p}+\beta} T D^{p}
$$

where $\alpha$ and $\beta$ are the gradient and intercept of the linear function and they have great influence on the approximation (equalization). On the other hand, $\gamma$ is the adjusting constant as well as the conventional method and it does not influence on the equalization. These are empirically determined through examinations of the relation between the number of sampled data and the optimal threshold.

2) Equalization using nonlinear approximation: On the other hand, the relation between the number of sampled data and the DP distance could be fitted by a nonlinear function as the broken line (b) in Fig. 6. The DP distances are adjusted by using the exponential function as

$$
T D_{e q}^{p}=\frac{\gamma}{\exp \left(\alpha \cdot S_{n u m}^{p}+\beta\right)} T D^{p}
$$

where $\alpha$ and $\beta$ are also constants for fitting the nonlinear function to the relation between the number of sampled data and the DP distance.

\section{B. Experiments}

In order to verify the effectiveness of the proposed threshold equalizing methods, we examined them in verification experiments using the SVC2004, again. The conditions are similar with those in Sect. III-B. The number of partitions in DP matching was 4.

Constants for approximation: $\alpha$ and $\beta$ were respectively 10 and -293 in the linear case and 0.0069 and 3.3 in the nonlinear case, which correspond to the solid and the broken lines in Fig. 6. From a viewpoint of their universality, it was better to determine them using a training data set, which was independent of a test data set. However, proposed equalizing methods are based on rough approximation of relation between the numbers of sampled data and optimal thresholds in the SVC2004. If the relation in the training data set is equivalent with that in the test data set, the proposed methods does not depend on the data used. On the other hand, the approximation depends on databases. The larger the number of data becomes, the more universal the constants. In both cases, $\gamma$ was set to a value which adjusts thresholds to around 2000.

The distribution of optimal thresholds after equalization is compared with that before equalization in Figs. 7 and 8. Uncolored triangles are before equalization and black ones are after equalization. It is confirmed that the optimal thresholds were adjusted to around 2000 and the variation range of the optimal thresholds was narrowed.

For making quantitative evaluation, we analyzed statistical variance of optimal threshold values. The variance before equalization was 0.27 but after equalization it was reduced to 0.05 in the linear case and 0.07 in the nonlinear case. The effect of threshold equalization was quantitatively confirmed.

Finally, EERs and statistical variances of optimal threshold values in various methods are summarized in Table II. Comparing the EER in the 4 partitioned DP matching with that in the normalized DP matching, it is confirmed that the proposed simply partitioning is more effective. Similarly, the proposed new threshold equalization methods are confirmed to be more efficient than the normalized DP matching and the conventional method. Moreover, combining the simply partitioned DP matching with the new threshold equalization is much more effective for improving the verification performance. Especially, the smallest EER of $14.6 \%$ was achieved when the threshold equalization using the linear approximation was applied. As confirmed in Fig. 8, the adjustment in the nonlinear case might be excessive when 


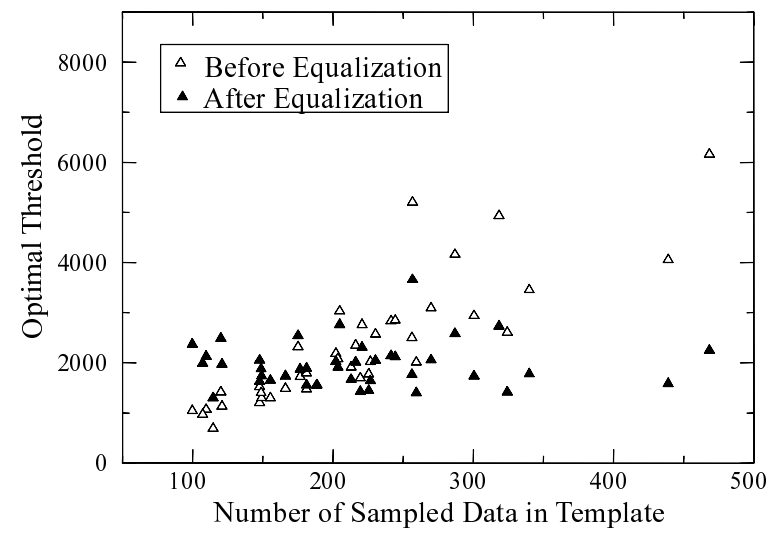

Figure 7. Distribution of optimal thresholds after equalization in the linear case.

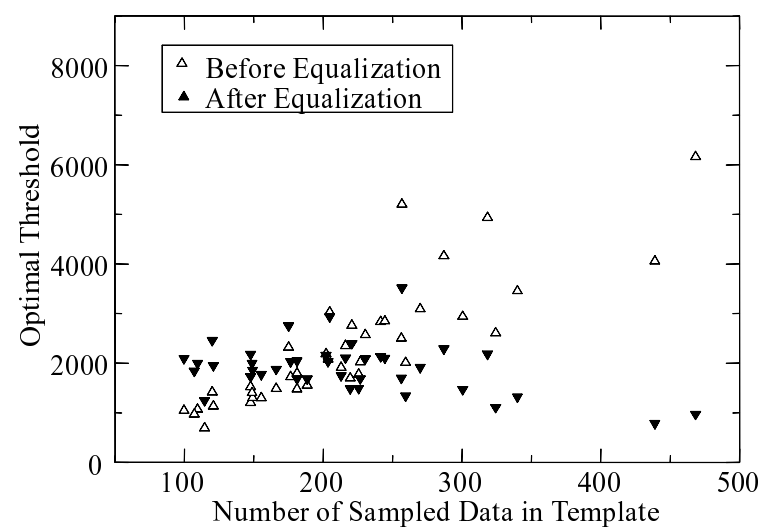

Figure 8. Distribution of optimal thresholds after equalization in the nonlinear case.

the number of sampled data were large. It is a future problem to adopt other nonlinear functions for approximating the relation between the number of sampled data and the optimal thresholds.

\section{CONCLUSiOnS}

In order to improve the performance of the DWT domain on-line signature verification, we introduced simply partitioned DP matching and threshold equalizing methods into the verification process. In the simply partitioned DP matching, accumulative errors were initialized at the beginning of the next partition; therefore, the DP distance was prevented from becoming excessively large. The threshold equalizing suppressed the variation range of optimal thresholds for all signatures; therefore, it prevented the verification performance from degrading by using a common threshold for all signatures. Especially, we proposed two equalization methods based on linear and nonlinear approximation of the relation between the number of sampled data and the optimal thresholds in signatures.
Table II

EERS AND STATISTICAL VARIANCES IN VARIOUS METHODS.

\begin{tabular}{|c|c|c|}
\hline Method & EER(\%) & Variance \\
\hline \hline Unnormalized DP & 25.4 & 0.54 \\
\hline Normalized DP & 20.0 & 0.17 \\
\hline 4 partitioned DP & 16.6 & 0.05 \\
\hline Conventional Equalization & 19.9 & 0.24 \\
\hline Linear Equalization & 19.0 & 0.22 \\
\hline Nonlinear Equalization & 19.5 & 0.23 \\
\hline $\begin{array}{c}\text { 4 partitioned DP } \\
\text { + Linear Equalization }\end{array}$ & 14.6 & 0.05 \\
\hline $\begin{array}{c}\text { 4 partitioned DP } \\
\text { + Nonlinear Equalization }\end{array}$ & 14.9 & 0.07 \\
\hline
\end{tabular}

In experiments using part of the signature database: SVC2004, the EER of about 15\% was obtained using the proposed simply partitioned DP matching and threshold equalizing, and it was improvement of about 5\% comparing with the performance using the general-used DP matching.

Distribution of optimal thresholds is not yet fully narrowed as shown in the experimental results; therefore, further improvement of threshold equalization is a problem to be studied.

\section{REFERENCES}

[1] A. Jain, R. Bolle and S. Pankanti, BIOMETRICS Personal Identification in Networked Society, Kluwer Academic Publishers, Massachusetts, 1999.

[2] I. Nakanishi, N. Nishiguchi, Y. Itoh, and Y. Fukui, "OnLine Signature Verification Based on Discrete Wavelet Domain Adaptive Signal Processing," Proc. of ICBA2004, pp. 584-591, Jul. 2004.

[3] I. Nakanishi, N. Nishiguchi, Y. Itoh, and Y. Fukui, "OnLine Signature Verification Based on Subband Decomposition by DWT and Adaptive Signal Processing," Electronics and Communications in Japan, Part 3, vol. 88, no. 6, pp. 1-11, Jun. 2005.

[4] I. Nakanishi, H. Sakamoto, Y. Itoh, and Y. Fukui, "Threshold Equalization for On-Line Signature Verification," IEICE Trans. Fundamentals, vol. E91-A, no. 8, pp. 2244-2247, Aug. 2008.

[5] S. Koike, I. Nakanishi, and Y. Itoh, "Threshold Equalization Methods for On-Line Signature Verification," Proc. of the 11th International Conference on Frontiers in Handwriting Recognition (ICFHR2008), Aug. 2008.

[6] M. Yoshimura and I. Yoshimura, "An Off-Line Verification Method for Japanese Signatures Based on a Sequential Application of Dynamic Programming Matching Method (in Japanese)," IEICE Trans. Information and Systems, vol. J81D-II, no. 10, pp. 2259-2266, Oct. 1998.

[7] T. Sano, N. Wada, T. Yoshida, and S. Hangai, "A Study on Segmentation Scheme for DP Matching in Japanese Signature Verification (in Japanese)," Proc. of 2007 IEICE General Conference, B-18-4, Mar. 2007.

[8] http://www.cse.ust.hk/svc2004/index.html 